\title{
Template Matching as a Tool to Analyze Electron Tomograms
}

\author{
R.H.M. Schoenmakers, ${ }^{*}$ A. Yakushevska, ${ }^{*}$ M.M.H. Storms, ${ }^{*}$ \\ * FEI Company, Achtseweg Noord 5, 5600 KK, Eindhoven, The Netherlands
}

Fundamental research within the scope of cell biology, structural biology and nano-technology is increasingly focusing on unraveling interactive biological and biochemical processes and pathways at the macromolecular level. For this, high resolution transmission electron microscopy (TEM) is indispensable. Of paramount importance is the three-dimensional visualization of macromolecular structures and molecular machines in their native hydrated state.

In addition, for optimal control of developmental and manufacturing processes of polymers, food and personal care products, exploration of product components or hybrids and their in vivo behavior at the macromolecular scale is indispensable. In order to observe biological structures as close as possible to their natural state, cryo-fixation is the indispensable method to preserve the specimen.

Single Particle Analysis (SPA) and tomography provide complimentary information. SPA can deliver high resolution images of a molecular complex, but it is taken out of its biological context. Cryo-electron Tomography (CET) can obtain information on the position of a complex, but the resolution of individual particles is limited. Template matching is a technique that has been explored for some time now as an indispensable tool to bridge the gap between the two regimes [e.g. 1-3]. However, due to the large computational demands of the technique, requiring it to run on clusters or supercomputers, its use is not yet widespread and has also hampered further exploration of the use of the technique for tomogram segmentation beyond the work of [4] or use in other, non-biological areas like nano-particle research.

In order to bridge this gap, FEI has developed the ARGOS software (Automated Recognition of Geometries, Objects and Segmentations). Based on massive parallel computations of high end GPUs (Graphical processing units, or video cards), it brings the speed of a medium sized CPU cluster to a single desktop PC. With this software it is possible to average of nano-particles or macromolecules found in a single or multiple tomograms to obtain higher resolution (especially for molecules which cannot easily be purified, e.g. membrane proteins), automatically finding macromolecules in their crowded native cellular context and relating orientation properties of these molecules to their environment. In addition, the produced correlation data can be used for guided, more quantitative segmentation purposes.

\section{References}

[1] Frangakis, A.S. et al., Proc Natl Acad Sci USA 99 (2002) 14153

[2] Nickell, S. et al., Nat Rev Mol Cell Biol 7 (2006), 225

[3] Ortiz, J.O. et al., J. Struct.Biol. 156 (2006) 334.

[4] Lebbink, M.N., PhD Thesis, University of Utrecht, The Netherlands (2009)

[5] We gratefully acknowledge Dr. Stephan Nickell (MPI Martinsried, Germany) and the Bram Koster group (LUMC, The Netherlands) for their assistance during the development of the ARGOS application. 